Pacific

Journal of

Mathematics

\title{
A FAMILY OF ISOCHRONOUS FOCI WITH DARBOUX FIRST INTEGRAL
}

JAUME GiNÉ AND JAUME LLIBRE 


\title{
A FAMILY OF ISOCHRONOUS FOCI WITH DARBOUX FIRST INTEGRAL
}

\author{
JAUME GinÉ AND JAUME LLIBRE
}

\begin{abstract}
We consider the class of polynomial differential equations $\dot{\boldsymbol{x}}=\lambda \boldsymbol{x}-\boldsymbol{y}+$ $P_{n}(x, y)+P_{2 n-1}(x, y), \dot{y}=x+\lambda y+Q_{n}(x, y)+Q_{2 n-1}(x, y)$ with $n \geq 2$, where $P_{i}$ and $Q_{i}$ are homogeneous polynomials of degree $i$. These systems have a focus at the origin if $\lambda \neq 0$, and have either a center or a focus if $\lambda=0$. Inside this class we identify a new subclass of Darboux integrable systems having either a focus or a center at the origin. Under generic conditions such Darboux integrable systems can have at most two limit cycles, and when they exist are algebraic. For the case $n=2$ and $n=3$ we present new classes of Darboux integrable systems having a focus.
\end{abstract}

\section{Introduction and statement of the results}

Three of the main problems in the qualitative theory of real planar differential systems are the determination of centers, limit cycles and first integrals. This paper deals mainly with the determination of first integrals and limit cycles.

As usual a center is a singular point having a neighborhood filled of periodic orbits, and a focus is a singular point having a neighborhood where all the orbits spiral in forward or in backward time to it.

Here we study real planar polynomial differential systems of the form

$$
\begin{aligned}
& \dot{x}=\lambda x-y+P_{n}(x, y)+P_{2 n-1}(x, y), \\
& \dot{y}=x+\lambda y+Q_{n}(x, y)+Q_{2 n-1}(x, y),
\end{aligned}
$$

where $P_{i}$ and $Q_{i}$ are homogeneous polynomials of degree $i$. Inside this class we will characterize a new subclass of Darboux integrable systems having either a focus or a center at the origin.

Giné is partially supported by MCYT grant BFM 2002-04236-C02-01, by a University of Lleida Project P01 and by DURSI of Government of Catalonia's Acció Integrada ACI2001-26. Llibre is partially supported by DGICYT grant BFM 2002-04236-C02-02 and by CICYT grant 2001SGR00173. MSC2000: 34C35, 34D30.

Keywords: integrability, algebraic limit cycle, focus, center. 
We establish some notation and preliminary results. In polar coordinates $(r, \theta)$, defined by

$$
x=r \cos \theta, \quad y=r \sin \theta,
$$

system (1) becomes

$$
\begin{aligned}
& \dot{r}=\lambda r+f_{n+1}(\theta) r^{n}+f_{2 n}(\theta) r^{2 n-1}, \\
& \dot{\theta}=1+g_{n+1}(\theta) r^{n-1}+g_{2 n}(\theta) r^{2 n-2},
\end{aligned}
$$

where

$$
\begin{aligned}
& f_{i}(\theta)=\cos \theta P_{i-1}(\cos \theta, \sin \theta)+\sin \theta Q_{i-1}(\cos \theta, \sin \theta), \\
& g_{i}(\theta)=\cos \theta Q_{i-1}(\cos \theta, \sin \theta)-\sin \theta P_{i-1}(\cos \theta, \sin \theta)
\end{aligned}
$$

are certain homogeneous trigonometric polynomials in the variables $\cos \theta$ and $\sin \theta$ having degree in the set $\{i, i-2, i-4, \ldots\}$. Indeed, $f_{i}(\theta)$ can be of the form $\left(\cos ^{2} \theta+\sin ^{2} \theta\right)^{s} f_{i-2 s}$ with $f_{i-2 s}$ a trigonometric polynomial of degree $i-2 s \geq 0$, and a similar situation occurs for $g_{i}(\theta)$.

If we impose $g_{n+1}(\theta)=g_{2 n}(\theta)=0$ and make the change $R=r^{n-1}$, system (3) becomes the differential equation

$$
\frac{d R}{d \theta}=(n-1)\left(\lambda R+f_{n+1}(\theta) R^{2}+f_{2 n}(\theta) R^{3}\right) .
$$

Differential equations of this kind appeared in Abel's studies on the theory of elliptic functions. For more details on Abel differential equations, see [Kamke 1943; Cheb-Terrab and Roche 2003; Gasull and Llibre 1990].

We say that all polynomial differential systems (1) with $g_{n+1}(\theta)=g_{2 n}(\theta)=0$ define class $\mathscr{F}$ if $f_{2 n}(\theta)$ and $f_{n+1}(\theta)$ satisfy

$$
f_{2 n}^{\prime}(\theta) f_{n+1}(\theta)-f_{2 n}(\theta) f_{n+1}^{\prime}(\theta)=(n-1)\left(a f_{n+1}(\theta)^{3}-\lambda f_{n+1}(\theta) f_{2 n}(\theta)\right)
$$

for some $a \in \mathbb{R}$. Clearly, the class of our polynomial differential systems (1) has dimension $6 n+4$ in the space of all coefficients, and the subclass $\mathscr{F}$ is an algebraic subvariety of it.

We shall prove that all polynomial differential systems (1) in class $\mathscr{F}$ have a Darboux first integral. We have found the subclass $\mathscr{F}$ thanks to the Abel differential equations studied in [Kamke 1943, pp. 24-25, cases (a-d)]. Using these same techniques new Darboux integrable systems are found in [Giné and Llibre 2004] for polynomial systems formed by a linear part plus homogeneous nonlinearities.

A function of the form $f_{1}^{\lambda_{1}} \ldots f_{p}^{\lambda_{p}} \exp (h / g)$, where $f_{i}, g$ and $h$ are polynomials in $\mathbb{C}[x, y]$ and the $\lambda_{i}$ 's are complex numbers, is called a Darboux function. System (1) is called Darboux integrable if the system has a first integral or an integrating factor which is a Darboux function (for a definition of a first integral and of an 
integrating factor, see [Chavarriga et al. 1999; Christopher and Llibre 2000], for instance). Our main result is the following:

Theorem 1. For polynomial differential systems (1) in the class $\mathscr{F}$ the following statements hold.

(a) If $\lambda \neq 0$ and $f_{2 n}(\theta) f_{n+1}(\theta) \neq 0$, then the origin is a focus and the system has the Darboux first integral $\tilde{H}(x, y)$ obtained from

$$
H(R, \theta)= \begin{cases}\frac{R \exp ((n-1) \lambda \theta) \exp \left(-\frac{1}{\sqrt{4 a-1}} \arctan \frac{\left(1+2 R f_{2 n}(\theta) / f_{n+1}(\theta)\right)}{\sqrt{4 a-1}}\right)}{\sqrt{R^{2} f_{2 n}^{2}(\theta) / f_{n+1}^{2}(\theta)+R f_{2 n}(\theta) / f_{n+1}(\theta)+a}} & \text { if } a>\frac{1}{4}, \\ \frac{R \exp ((n-1) \lambda \theta) \exp \frac{1}{1+2 R f_{2 n}(\theta) / f_{n+1}(\theta)}}{1+2 R f_{2 n}(\theta) / f_{n+1}(\theta)} & \text { if } a=\frac{1}{4}, \\ \frac{R \exp ((n-1) \lambda \theta)\left(\sqrt{1-4 a}+1+\frac{2 R f_{2 n}(\theta)}{f_{n+1}(\theta)}\right)^{(-1+1 / \sqrt{1-4 a}) / 2}}{\left(\sqrt{1-4 a}-1-\frac{2 R f_{2 n}(\theta)}{f_{n+1}(\theta)}\right)^{(1+1 / \sqrt{1-4 a}) / 2}} & \text { if } a<\frac{1}{4}, a \neq 0, \\ \frac{\exp ((n-1) \lambda \theta) f_{2 n}(\theta)}{f_{n+1}(\theta)} & \text { if } a=0,\end{cases}
$$

through the changes of variables (2) and with $R=r^{n-1}$.

(b) If $\lambda \neq 0$ and $a=f_{2 n}(\theta) f_{n+1}(\theta)=0$, then the origin is a focus and the system has the Darboux first integral $\tilde{H}(x, y)$ obtained from

$H(R, \theta)= \begin{cases}\frac{\exp ((n-1) \lambda \theta)}{R}+(n-1) \int \exp ((n-1) \lambda \theta) f_{n+1}(\theta) d \theta & \text { if } f_{2 n}(\theta)=0, \\ \frac{\exp (2(n-1) \lambda \theta)}{R^{2}}+2(n-1) \int \exp (2(n-1) \lambda \theta) f_{2 n}(\theta) d \theta & \text { if } f_{n+1}(\theta)=0,\end{cases}$ through the changes of variables (2) and with $R=r^{n-1}$.

(c) If $\lambda=0$, the origin is a center, and the system has an analytic first integral $\tilde{H}(x, y)$ obtained by taking $\lambda=0$ in the expressions for $\tilde{H}(x, y)$ in (a) and (b).

(d) If $\lambda=0$, the origin is a center, and the following systems have a rational first integral:

(d1) Systems with $f_{n+1}(\theta)=f_{2 n}(\theta)=0$.

(d2) Systems with $f_{2 n}(\theta)=0$ and $\int_{0}^{2 \pi} f_{n+1}(\theta) d \theta=0$.

(d3) Systems with $f_{n+1}(\theta)=0$ and $\int_{0}^{2 \pi} f_{2 n}(\theta) d \theta=0$.

(d4) Systems whose a (defined in (5)) satisfies $a<\frac{1}{4}, a \neq 0$, and $\sqrt{1-4 a}$ is rational.

Theorem 1 will be proved in Section 2. Part (c) follows easily from (a) and (b).

A limit cycle of system (1) is a periodic orbit isolated in the set of periodic orbits of system (1). We say that a limit cycle $\gamma$ is algebraic if it is contained in an algebraic curve. 
Theorem 2. If a system (1) in class $\mathscr{F}$ with $\lambda \neq 0$ and $f_{2 n}(\theta) f_{n+1}(\theta) \neq 0$ has a limit cycle, it is algebraic. Moreover, such a system can have at most two limit cycles. There are systems with 0,1 or 2 limit cycles.

In the course of the proof, given in Section 3, we provide an explicit expression for algebraic limit cycles.

Systems (1) for $n=2$ are cubic differential systems. The problem of determining when a cubic differential system (1) has a center at a singular point is open. Trying to distinguish whether a weak focus of a general cubic system is a center or a focus has produced disappointing results, due to the huge expressions obtained for its Poincaré-Liapunov constants; see [Schlomiuk 1993]. But several authors have studied particular subclasses of cubic polynomial differential systems; see for instance [Pearson et al. 1996] and the references therein. The center problem for cubic polynomial differential system (1) satisfying $x Q_{3}(x, y)-y P_{3}(x, y)=0$ has been totally solved in [Chavarriga and Giné 1998; Lloyd et al. 1997].

Other polynomial differential systems recently investigated are those of the form

$$
\dot{x}=y+x F(x, y), \quad \dot{y}=-x+y F(x, y),
$$

where $F(x, y)=\sum_{i=1}^{4} F_{i}(x, y)$ for homogeneous polynomials $F_{i}(x, y)$ of degree $i$. Such systems satisfy $x Q_{i}(x, y)-y P_{i}(x, y)=0$ for $i=1, \ldots, 4$; therefore, they have constant angular speed $\dot{\theta}=1$. When a system (6) has a center at the origin, this center is called a uniformly isochronous center [Conti 1994]. If $F(x, y)=0$, the origin is a linear center. The conditions for a system (6) to have a center have been studied in [Collins 1997] when $F_{3}=F_{4}=0$. Systems of the form (6) have been studied in [Giné and Santallusia 2001] in the case that $F(x, y)$ is of degree 3 with $F_{2}=0$, and in [Chavarriga et al. 2001] in the case that $F(x, y)$ is of degree 3 . The case where $F(x, y)$ is of degree 4 is totally solved in [Volokitin 2002] when $F_{1}=F_{3}=0$.

It is easy to check that systems (1) with $n=2$ satisfying $g_{3}(\theta)=g_{4}(\theta)=0$ can be written into the form

$$
\begin{aligned}
& \dot{x}=\lambda x-y+x\left(\alpha x+\beta y+A x^{2}+B x y+C y^{2}\right), \\
& \dot{y}=x+\lambda y+y\left(\alpha x+\beta y+A x^{2}+B x y+C y^{2}\right),
\end{aligned}
$$

where $\alpha, \beta, A, B$ and $C$ are arbitrary constants. In [Collins 1997] it has been proved that the origin of system (7) is a center if and only if

$$
\lambda=0, \quad A+C=0 \quad \text { and } \quad A \alpha^{2}+B \alpha \beta+C \beta^{2}=0 .
$$

In Corollary 5 we compute the class of cubic polynomial differential systems satisfying (a) and (b) in Theorem 1, thereby exhibiting new classes of Darboux integrable cubic systems having a focus. 
Polynomial systems (1) with $n=3$ satisfying $g_{4}(\theta)=g_{6}(\theta)=0$ can be written to the form

$$
\begin{aligned}
& \dot{x}=\lambda x-y+x\left(A x^{2}+B x y+C y^{2}+D x^{4}+E x^{3} y+F x^{2} y^{2}+G x y^{3}+H y^{4}\right), \\
& \dot{y}=x+\lambda y+y\left(A x^{2}+B x y+C y^{2}+D x^{4}+E x^{3} y+F x^{2} y^{2}+G x y^{3}+H y^{4}\right),
\end{aligned}
$$

where $A, B, C, D, E, F, G$ and $H$ are arbitrary constants. Volokitin [2002] has proved that the origin of system (8) is a center if and only if one of the following sets of conditions are satisfied:

(i) $\lambda=0, A=B=C=0$, and $F=-3(D+H)$.

(ii) $\lambda=0$ and $A=C=D=F=H=0$.

(iii) $\lambda=0, A \neq 0, C=-A, F=3 B(A E-B D) / 2 A^{2}$,

$$
H=\frac{-2 A^{2} D+B(B D-A E)}{2 A^{2}}, \quad G=\frac{2 A^{2} B D+\left(2 A^{2}-B^{2}\right)(B D-A E)}{2 A^{3}} .
$$

In Corollary 6 we will provide new classes of Darboux integrable systems (8) having either a focus or a center at the origin.

\section{Proof of Theorem 1}

Proof of Theorem 1(a). Following [Kamke 1943, p. 25, case (d)], we make the change of variables $(R, \theta) \rightarrow(\eta, \xi)$ defined by $R=u(\theta) \eta(\xi)$, where $u(\theta)=$ $\exp ((n-1) \lambda \theta)$ and $\xi=\int \exp ((n-1) \lambda \theta)(n-1) f_{n+1}(\theta) d \theta$. This transformation writes the Abel differential equation (4) into the form

$$
\eta^{\prime}(\xi)=g(\xi) \eta(\xi)^{3}+\eta(\xi)^{2},
$$

where $g(\xi)=\exp ((n-1) \lambda \theta) f_{2 n}(\theta) / f_{n+1}(\theta)$ and ${ }^{\prime}=d / d \xi$. Making the change $\xi \rightarrow t$ in the independent variable defined by $\xi^{\prime}=-1 /(t \eta(\xi))$, where now ${ }^{\prime}=d / d t$, equation (9) becomes

$$
t^{2} \xi^{\prime \prime}(t)+g(\xi(t))=0
$$

Note that $g(\xi)=a \xi$ means

$$
\exp ((n-1) \lambda \theta) f_{2 n}(\theta) / f_{n+1}(\theta)=a \int \exp ((n-1) \lambda \theta)(n-1) f_{n+1}(\theta) d \theta
$$

equivalently, by differentiating with respect to $\theta$, we get

$$
\frac{d}{d \theta} \frac{f_{2 n}(\theta)}{f_{n+1}(\theta)}=a(n-1) f_{n+1}(\theta)-\frac{(n-1) \lambda f_{2 n}(\theta)}{f_{n+1}(\theta)},
$$

which is equivalent to condition (5). Thus $g(\xi)=a \xi$, and (10) is an Euler differential equation. Applying the change $t=\exp (\tau)$ to the independent variable, 
equation (10) then becomes a linear ordinary differential equation with constant coefficients:

$$
\xi^{\prime \prime}(\tau)-\xi^{\prime}(\tau)+a \xi(\tau)=0
$$

where ${ }^{\prime}=d / d \tau$. Equation (12) has the characteristic equation $k^{2}-k+a=$ 0 , so its general solution is $\xi(\tau)=C_{1} \exp (\tau / 2)+C_{2} \tau \exp (\tau / 2)$ if $a=\frac{1}{4}$, and $\xi(\tau)=C_{1} \exp \left(k_{1} \tau\right)+C_{2} \exp \left(k_{2} \tau\right)$ if $a \neq \frac{1}{4}$, where $k_{1}$ and $k_{2}$ are the roots of the characteristic equation. Going back to the independent variable $t=\exp (\tau)$, the solution of the Euler differential equation is $\xi(t)=C_{1} \sqrt{t}+C_{2} \sqrt{t} \ln t$ if $a=\frac{1}{4}$ and $\xi(t)=C_{1} t^{k_{1}}+C_{2} t^{k_{2}}$ if $a \neq \frac{1}{4}$.

Finally, going back through the change of variables to the variables $(R, \theta)$ and taking into account whether the roots $k_{1}$ and $k_{2}$ are real or complex, we obtain the first integrals shown in statement (a), according to the value of $a$.

We now prove that the systems in (a) are Darboux integrable, by showing that all terms that appear in the first integral of those systems are of the form $f_{1}^{\lambda_{1}} \ldots f_{p}^{\lambda_{p}}$, with $f_{i}$ a polynomial and $\lambda_{i}$ a complex number. First, the term $\exp ((1-n) \lambda \theta)$ takes the form

$$
\begin{aligned}
\exp ((1-n) \lambda \theta) & =\exp ((1-n) \lambda \arctan (y / x)) \\
& =(x+i y)^{i(n-1) \lambda / 2}(x-i y)^{-i(n-1) \lambda / 2} .
\end{aligned}
$$

Recall that if $f=0$, with $f \in \mathbb{C}[x, y]$, is an invariant algebraic curve of a real polynomial differential system, the complex conjugate $\bar{f}=0$ is also an invariant algebraic curve; see [Christopher and Llibre 2000], for instance. Therefore, if among the invariant algebraic curves of system (1) there occurs a complex conjugate pair $f=0$ and $\bar{f}=0$, the first integral has a factor of the form $f^{\mu} \bar{f}^{\bar{\mu}}$, which is the (multivalued) real function

$$
\left((\operatorname{Re} f)^{2}+(\operatorname{Im} f)^{2}\right)^{\operatorname{Re} \mu} \exp \left(-2 \operatorname{Im} \mu \arctan \frac{\operatorname{Im} f}{\operatorname{Re} f}\right) .
$$

On the other hand, writing $R=r^{n-1}$, it follows that

$$
F=\frac{f_{n+1}(\theta)+2 R f_{2 n}(\theta)}{f_{n+1}(\theta)}=\frac{r^{n+1}\left(f_{n+1}(\theta)+2 r^{n-1} f_{2 n}(\theta)\right)}{r^{n+1} f_{n+1}(\theta)},
$$

is a rational function in cartesian coordinates because $f_{2 n}(\theta)$ and $f_{n+1}(\theta)$ are homogeneous trigonometric polynomials of degree $2 n$ and $n+1$, respectively. Taking into account these relations, the first integral for $a>\frac{1}{4}$ is the Darboux function

$$
H(\rho, \theta)=\rho \exp ((1-n) \lambda \theta) f^{\mu} \bar{f}^{\bar{\mu}},
$$


where $\operatorname{Re} f=F, \operatorname{Im} f=\sqrt{4 a-1}, \operatorname{Re} \mu=-\frac{1}{2}$, and $\operatorname{Im} \mu=1 /(2 \sqrt{4 a-1})$. The first integral for $a=\frac{1}{4}$ is the Darboux function

$$
H(\rho, \theta)=\rho \exp ((1-n) \lambda \theta) \exp (1 / F) / F .
$$

The first integral for $a<\frac{1}{4}$ and $a \neq 0$ is the Darboux function

$$
H(\rho, \theta)=\rho \exp ((1-n) \lambda \theta)|\sqrt{1-4 a}+F|^{\mu_{1}}|\sqrt{1-4 a}-F|^{\mu_{2}},
$$

where $\mu_{1}=\frac{1}{2}(-1+1 / \sqrt{1-4 a})$ and $\mu_{2}=\frac{1}{2}(1+1 / \sqrt{1-4 a})$. Finally, the first integral for $a=0$ is the Darboux function

$$
H(\rho, \theta)=\frac{\exp ((1-n) \lambda \theta) f_{2 n}(\theta) r^{2 n}}{r^{n-1} f_{n+1}(\theta) r^{n+1}},
$$

and this completes the proof of statement (a).

Proof of Theorem 1(b). In the cases $f_{2 n}(\theta)=0$ and $f_{n+1}(\theta)=0$, the Abel differential equation (4) is the Bernoulli differential equation $d R / d \theta=(n-1)\left(f_{n+1}(\theta) R^{2}+\right.$ $\lambda R)$ and $d R / d \theta=(n-1)\left(f_{2 n}(\theta) R^{3}+\lambda R\right)$, respectively. Solving these Bernoulli equations we obtain the first integrals of statement (b).

Systems of statement (b) are Darboux integrable because their first integrals are obtained by integrating elementary functions; see [Singer 1992] for more details. The integrals appearing in the first integrals in question can be computed using recurrence formulas; see for instance [Petit Bois 1961, p. 149].

Proof of Theorem 1(c). The proof follows easily taking $\lambda=0$ in statements (a) and (b).

Proof of Theorem 1(d). If $f_{n+1}(\theta)=f_{2 n}(\theta)=0$, system (3) with $\lambda=0$ satisfies $\dot{r}=0$ and therefore it has a polynomial first integral $H=x^{2}+y^{2}$. Statement (d1) follows.

If $f_{2 n}(\theta)=0$, from the Abel differential equation (4) it is easy to derive that $H(R, \theta)=1 / R+(n-1) \int f_{n+1}(\theta) d \theta$ is a first integral. Taking into account that $\int_{0}^{2 \pi} f_{n+1}(\theta) d \theta$ vanishes and going back to cartesian variables, we obtain a rational first integral and (d2) follows.

If $f_{n+1}(\theta)=0$, again from the Abel differential equation (4) it is easy to derive that $H(R, \theta)=1 / R^{2}+2(n-1) \int f_{2 n}(\theta) d \theta$ is a first integral. Taking into account that $\int_{0}^{2 \pi} f_{2 n}(\theta) d \theta$ vanishes and going back to cartesian variables, we obtain a rational first integral, and (d3) follows.

Finally, from the expression of the first integral $H(R, \theta)$ for $a<\frac{1}{4}$ and $a \neq 0$ with $\sqrt{1-4 a}$ rational, we have $H^{2}(R, \theta)=R^{2}|\sqrt{1-4 a}+F|^{2 \mu_{1}}|\sqrt{1-4 a}-F|^{2 \mu_{2}}$, where $\mu_{1}$ and $\mu_{2}$ are defined at the end of the proof of part (a). Therefore, a convenient power of $H^{2}(R, \theta)$ gives a rational first integral. There follows (d4). 
Now we investigate whether it is possible to find other integrable classes from the well known integrable cases of the Abel differential equation. Following [Kamke 1943 , p. 24, case (4)], first we perform the change of variables $(R, \theta) \rightarrow(\eta, \xi)$ defined by $R=w(\theta) \eta(\xi)-f_{n+1}(\theta) /\left(3 f_{2 n}(\theta)\right)$, where

$$
w(\theta)=\exp \left(\int(n-1)\left(\lambda-f_{n+1}^{2}(\theta) /\left(3 f_{2 n}(\theta)\right)\right) d \theta\right)
$$

and $\xi=\int(n-1) f_{2 n}(\theta) w^{2}(\theta) d \theta$. This puts the Abel equation (4) into the normal form

$$
\eta^{\prime}(\xi)=\eta(\xi)^{3}+I(\theta)
$$

where

$$
I(\theta)=\frac{1}{(n-1) f_{2 n}(\theta) w^{3}(\theta)}\left(\frac{d}{d \theta} \frac{f_{n+1}(\theta)}{3 f_{2 n}(\theta)}-\frac{(n-1) \lambda f_{n+1}(\theta)}{3 f_{2 n}(\theta)}+\frac{2(n-1) f_{n+1}^{3}(\theta)}{27 f_{2 n}^{2}(\theta)}\right) .
$$

From the definition of $w(\theta)$ we have

$$
\begin{aligned}
\ln |w(\theta)| & =(n-1) \int\left(\lambda-\frac{f_{n+1}^{2}(\theta)}{3 f_{2 n}(\theta)}\right) d \theta \\
& =(n-1) \int \frac{f_{n+1}(\theta)}{f_{2 n}(\theta)}\left(\frac{\lambda f_{2 n}(\theta)}{f_{n+1}(\theta)}-\frac{f_{n+1}(\theta)}{3}\right) d \theta .
\end{aligned}
$$

In the case $a \neq 0$, the right-hand side of (14) becomes, upon use of (5) (or, equivalently, of (11)),

$$
\begin{aligned}
-\frac{1}{3 a} \int \frac{\frac{d}{d \theta}\left(f_{2 n}(\theta) / f_{n+1}(\theta)\right)}{f_{2 n}(\theta) / f_{n+1}(\theta)} d \theta+ & \left(1-\frac{1}{3 a}\right)(n-1) \int \lambda d \theta \\
& =-\frac{1}{3 a} \ln \left|\frac{f_{2 n}(\theta)}{f_{n+1}(\theta)}\right|+\left(1-\frac{1}{3 a}\right)(n-1) \lambda \theta .
\end{aligned}
$$

This leads to $w(\theta)=\left|f_{2 n}(\theta) / f_{n+1}(\theta)\right|^{-1 / 3 a} \exp ((n-1)(1-1 /(3 a)) \lambda \theta)$, so $I(\theta)$ becomes

$$
I(\theta)=\left(\frac{2-9 a}{27}\right)\left(\frac{f_{2 n}(\theta)}{f_{n+1}(\theta)}\right)^{(1-3 a) / a} \exp ((n-1)(1-3 a) \lambda \theta / a) .
$$

It is easy to see that $I(\theta)=0$ for $a=\frac{2}{9}$ and $I(\theta)=-\frac{1}{27}$ for $a=\frac{1}{3}$. In these two cases, we can separate variables in the differential equation (13) and obtain the associated first integrals. But $I(\theta)=0$ and $I(\theta)=-\frac{1}{27}$ imply that (5) holds with $a=\frac{2}{9}$ and $a=\frac{1}{3}$, respectively. So we obtain cases already studied. New cases of integrability would only appear for $I(\theta) \neq 0,-\frac{1}{27}$. 
Cases (b) and (c) of the Abel differential equation of [Kamke 1943, p. 25] again lead to the case already studied, with $a=\frac{2}{9}$.

\section{Algebraic limit cycles with Darboux first integral}

The next proposition presents what is probably the easiest example of a polynomial differential system that has a Darboux first integral and an algebraic limit cycle. Other examples of this kind were given in [Dolov 1976; Kooij and Christopher 1993; Christopher 1994]. In fact, it has now been proved that any finite configuration of limit cycles is realizable by algebraic limit cycles of a Darboux integrable polynomial differential systems [Llibre and Rodríguez 2004].

Proposition 3 [Chavarriga et al. 1999]. The differential system

$$
x^{\prime}=x-y-x\left(x^{2}+y^{2}\right), \quad y^{\prime}=x+y-y\left(x^{2}+y^{2}\right)
$$

has the algebraic solution $x^{2}+y^{2}-1=0$ as a limit cycle. In polar coordinates (2) the function $H(r, \theta)=\left(r^{2}-1\right) \exp (2 \theta) / r^{2}=C$ is a Darboux first integral defined on $\mathbb{R}^{2} \backslash \Sigma$, where $\Sigma=\{(0,0)\} \cup\left\{(x, y): x^{2}+y^{2}-1=0\right\}$.

To study the existence or nonexistence of limit cycles in system (1) we shall use the following result.

Theorem 4 [Giacomini et al. 1996, Theorem 9]. Let $(P, Q)$ be a $C^{1}$ vector field defined in an open subset $U$ of $\mathbb{R}^{2}$. Let $V=V(x, y)$ be a $C^{1}$ solution of the linear partial differential equation

$$
P \frac{\partial V}{\partial x}+Q \frac{\partial V}{\partial y}=\left(\frac{\partial P}{\partial x}+\frac{\partial Q}{\partial y}\right) V
$$

defined in $U$. If $\gamma$ is a limit cycle of $(P, Q)$, then $\gamma$ is contained in $\{(x, y) \in U$ : $V(x, y)=0\}$.

Under the assumptions of Theorem 4, the function $1 / V$ is an integrating factor in $U \backslash\{V(x, y)=0\}$ (see [Chavarriga et al. 1999; Christopher and Llibre 2000] for details). So the function $V$ is called an inverse integrating factor.

Proof of Theorem 2. For systems (1) in class $\mathscr{F}$ with $\lambda \neq 0$ and $f_{2 n}(\theta) f_{n+1}(\theta) \neq 0$, it is easy to check that

$$
V(\rho, \theta)=R\left(R^{2} f_{2 n}^{2}(\theta) / f_{n+1}^{2}(\theta)+R f_{2 n}(\theta) / f_{n+1}(\theta)+a\right)
$$

is an inverse integrating factor of the associated Abel differential equation (4). Notice that $V$ is defined for all $(R, \theta)$ such that $f_{n+1}(\theta) \neq 0$. Again by Theorem 4, if system (1) and consequently its associated Abel equation (4) have limit cycles, those of the Abel equation must be contained in the set $\{V(R, \theta)=0\}$. 
From the expression of the inverse integrating factor, the unique possible limit cycles must be given by

$$
R(\theta)= \begin{cases}\frac{1}{2}(-1 \pm \sqrt{1-4 a}) f_{n+1}(\theta) / f_{2 n}(\theta) & \text { if } a<\frac{1}{4} \\ -\frac{1}{2} f_{n+1}(\theta) / f_{2 n}(\theta) & \text { if } a=\frac{1}{4} .\end{cases}
$$

For these expressions to define limit cycles, $R(\theta)$ must be defined and positive for all $\theta$. Since $f_{2 n}(\theta)$ and $f_{n+1}(\theta)$ are homogeneous trigonometric polynomials of degree $2 n$ and $n+1$ respectively, we conclude that $n$ must be odd and $a \leq \frac{1}{4}$.

Clearly a system in our class (1) has no limit cycles if $n$ is even, or if $a>\frac{1}{4}$ and $f_{2 n}(\theta) f_{n+1}(\theta) \neq 0$. It can have one limit cycle if $a=\frac{1}{4}$ : for instance, setting $A=2, B=E=0$ in Corollary $6(\mathrm{c})$ yields the system $\dot{x}=-y-x\left(x^{2}+y^{2}-1\right)^{2}$, $\dot{y}=x-y\left(x^{2}+y^{2}-1\right)^{2}$, which has exactly one limit cycle, the circle $x^{2}+y^{2}-1=0$. And the system can have two limit cycles if $n$ is odd and $a<\frac{1}{4}$ : setting $A=4$, $B=E=0$ in Corollary $6(\mathrm{c})$ yields the system $\dot{x}=x-y-x\left(x^{2}+y^{2}-2\right)^{2}$, $\dot{y}=x+y-y\left(x^{2}+y^{2}-2\right)^{2}$, which has exactly two limit cycles given, $x^{2}+y^{2}-3=0$ and $x^{2}+y^{2}-1=0$. This completes the proof.

\section{Some corollaries}

System (1) with $n=2$ and $g_{3}(\theta)=g_{4}(\theta)=0$ - i.e., the cubic system (7) has a focus or a center at the origin. The following corollary characterizes cubic polynomial systems (7) belonging to class $\mathscr{F}$.

Corollary 5. A cubic system (7) with $\lambda \neq 0$ belongs to class $\mathscr{F}$ if and only if one of the following statements holds.

(a) $\alpha=\beta=0$. Then (7) has the Darboux first integral

$$
H(x, y)=\frac{\left(x^{2}+y^{2}\right) \exp \left(-2 \lambda \arctan \frac{y}{x}\right)}{\mathscr{P}_{2}(x, y)},
$$

where $\mathscr{P}_{2}(x, y)=2 \lambda^{3}+(A+C)\left(x^{2}+y^{2}\right)+2 \lambda^{2}\left(A x^{2}+y(B x+C y)\right)+$ $\lambda\left(2-2 C x y+2 A x y+B\left(y^{2}-x^{2}\right)\right)$.

(b) $A=a \alpha(\alpha \lambda-\beta) /\left(1+\lambda^{2}\right), B=a\left(\alpha^{2}-\beta^{2}+2 \alpha \beta \lambda\right) /\left(1+\lambda^{2}\right)$, and $C=$ $a \beta(\alpha+\beta \lambda) /\left(1+\lambda^{2}\right)$. Then (7) has, if $a>\frac{1}{4}$, the Darboux first integral $H(x, y)=$

$$
\frac{\left(x^{2}+y^{2}\right) \exp \left(-2 \lambda \arctan \frac{y}{x}-\frac{2}{\sqrt{1-4 a}} \arctan \frac{1+\lambda^{2}-2 a(\beta-\alpha \lambda) x+2 a(\alpha+\beta \lambda) y}{\left(1+\lambda^{2}\right) \sqrt{1-4 a}}\right)}{\mathscr{P}_{2}(x, y)},
$$

where $\mathscr{P}_{2}(x, y)=1+k^{4}+a \beta^{2} x^{2}+\alpha y+a \alpha^{2} y^{2}+k^{3}(\alpha x+\beta y)+k(\alpha x+\beta y)(1-$ $2 a \beta x+2 a \alpha y)-\beta(x+2 a \alpha x y)+k^{2}\left(2+a \alpha^{2} x^{2}+\alpha y+a \beta^{2} y^{2}+\beta x(-1+2 a \alpha y)\right)$; 
if $a<\frac{1}{4}$ and $a \neq 0$ it has the Darboux first integral

$$
H(x, y)=\frac{\left(x^{2}+y^{2}\right) \exp \left(-2 \lambda \arctan \frac{y}{x}\right) \mathscr{R}_{1}(x, y)}{\mathscr{R}_{2}(x, y)},
$$

where

$\mathscr{R}_{1}(x, y)=\left((-1+\sqrt{1-4 a})\left(1+\lambda^{2}\right)-2 a(-\beta x+\alpha \lambda x+\alpha y+\beta \lambda y)\right)^{-1-1 / \sqrt{1-4 a}}$,

$\mathscr{R}_{2}(x, y)=\left((-1-\sqrt{1-4 a})\left(1+\lambda^{2}\right)+2 a(-\beta x+\alpha \lambda x+\alpha y+\beta \lambda y)\right)^{1-1 / \sqrt{1-4 a}} ;$

in the case $a=\frac{1}{4}$ the Darboux first integral is

$$
H(x, y)=\frac{\left(x^{2}+y^{2}\right) \exp \left(-2 \lambda \arctan \frac{y}{x}-\frac{2\left(1+\lambda^{2}\right)}{1+\lambda^{2}-2 a \beta x+2 a \alpha \lambda x+2 a(\alpha+\beta \lambda) y}\right)}{\left(1+\lambda^{2}-2 a \beta x+2 a \alpha \lambda x+2 a(\alpha+\beta \lambda) y\right)^{2}} ;
$$

and in the case $a=0$ the Darboux first integral is

$$
H(x, y)=\frac{\left(x^{2}+y^{2}\right) \exp \left(-2 \lambda \arctan \frac{y}{x}\right)}{\left(1+\lambda^{2}-\beta x+\alpha \lambda x+\alpha y+\beta \lambda y\right)^{2}} .
$$

Consequently, for $\lambda \neq 0$ these cubic systems have a focus at the origin and are Darboux integrable.

Proof. This follows from parts (a) and parts (b) of Theorem 1 for $n=2$, after tedious computations.

System (1) with $n=3$ and $g_{4}(\theta)=g_{6}(\theta)=0$-i.e., the quintic system (8)has a focus or a center at the origin. The following corollary characterizes quintic polynomial systems (8) belonging to class $\mathscr{F}$.

Corollary 6. A system (8) with $\lambda \neq 0$ belongs to class $\mathscr{F}$ if and only if one of the following statements holds.

(a) $A=B=C=0$. Then (8) has the Darboux first integral given by Theorem $1(b)$ with $n=3$ and $f_{4}(\theta)=0$.

(b) $A=B=D=E=0, F=a C^{2} /\left(2 \lambda\left(1+\lambda^{2}\right)\right), G=-a C^{2} /\left(1+\lambda^{2}\right)$ and $H=a C^{2}\left(1+2 \lambda^{2}\right) /\left(2 \lambda\left(1+\lambda^{2}\right)\right)$. Then (8) has the Darboux first integral given by Theorem $1(a)$ with $n=3$.

(c) $B=2 \lambda A, C=2 \lambda D\left(1+\lambda^{2}\right)-a A^{2} /(a A), E=2 a A^{2}, F=2 \lambda\left(2 a^{2} A^{4}+\right.$ $\left.D^{2}-a \lambda A^{2} D+\lambda^{2} D^{2}\right) /\left(a A^{2}\right), G=2\left(4 a \lambda A^{2} D-a^{2} A^{4}-2 \lambda^{2} D^{2}+4 a \lambda^{3} A^{2} D-\right.$ $\left.2 \lambda^{4} D^{2}\right) /\left(a A^{2}\right)$ and $H=D\left(1+2 \lambda^{2}\right)\left(2 \lambda D\left(1+\lambda^{2}\right)-a A^{2}\right) /\left(a A^{2}\right)$. Then (8) has the Darboux first integral given by Theorem $1(a)$ with $n=3$.

(d) $C=\left(-a A B+a \lambda B^{2}-2 a \lambda A^{2}+2 \lambda E-4 a \lambda^{2} A B+2 \lambda^{3} E\right) /(a(B-2 \lambda A))$, $D=A\left(2 a A^{2}-E\right) /(2 \lambda A-B), F=\left(3 a^{2} A B^{3}+8 \lambda a^{2} A^{4}-12 a^{2} \lambda A^{2} B^{2}-\right.$ $\left.8 a \lambda A^{2} E-a \lambda B^{2} E+2 \lambda E^{2}+16 a^{2} \lambda^{2} A^{3} B+2 a \lambda^{2} A B E-8 a \lambda^{3} A^{2} E+2 \lambda^{3} E^{2}\right) /$ 
$\left(a(B-2 \lambda A)^{2}\right), G=\left(a^{2} B^{4}-4 a^{2} A^{2} B^{2}+a B^{2} E-4 a^{2} \lambda A B^{3}+4 a \lambda A B E+\right.$ $\left.4 a \lambda^{2} A^{2} E-4 \lambda^{2} E^{2}+8 a \lambda^{3} A B E-4 \lambda^{4} E^{2}\right) /\left(a(B-2 \lambda A)^{2}\right), H=\left(-2 a A^{2}+\right.$ $\left.a B^{2}+E-4 a \lambda A B+2 \lambda^{2} E\right)\left(-a A B-2 a \lambda A^{2}+a \lambda B^{2}+2 \lambda E-4 a \lambda^{2} A B+\right.$ $\left.2 \lambda^{3} E\right) /\left(a(B-2 \lambda A)^{2}\right)$. Then (8) has the Darboux first integral given by Theorem 1(a) with $n=3$.

(e) $D=E=F=G=H=0$. Then (8) has the Darboux first integral given by Theorem $1(b)$ with $n=3$ and $f_{6}(\theta)=0$.

Consequently, for $\lambda \neq 0$ these quintic systems have a focus at the origin and are Darboux integrable.

Proof. This follows from parts (a) and parts (b) of Theorem 1 for $n=3$, after tedious computations using a computer-algebra program.

\section{References}

[Chavarriga and Giné 1998] J. Chavarriga and J. Giné, "Integrability of cubic systems with degenerate infinity”, Differential Equations Dynam. Systems 6:4 (1998), 425-438. MR 2001f:34052 Zbl 0998.34001

[Chavarriga et al. 1999] J. Chavarriga, H. Giacomini, J. Giné, and J. Llibre, "On the integrability of two-dimensional flows", J. Differential Equations 157:1 (1999), 163-182. MR 2000h:37058 Zbl 0940.37005

[Chavarriga et al. 2001] J. Chavarriga, I. A. García, and J. Giné, "On integrability of differential equations defined by the sum of homogeneous vector fields with degenerate infinity", Internat. J. Bifur. Chaos Appl. Sci. Engrg. 11:3 (2001), 711-722. MR 2002g:34062

[Cheb-Terrab and Roche 2003] E. S. Cheb-Terrab and A. D. Roche, "An Abel ordinary differential equation class generalizing known integrable classes", European J. Appl. Math. 14:2 (2003), 217229. MR 2004c:34003 Zbl 1046.34004

[Christopher 1994] C. J. Christopher, "Invariant algebraic curves and conditions for a centre", Proc. Roy. Soc. Edinburgh Sect. A 124:6 (1994), 1209-1229. MR 95m:34052 Zbl 0821.34023

[Christopher and Llibre 2000] C. Christopher and J. Llibre, "Integrability via invariant algebraic curves for planar polynomial differential systems", Ann. Differential Equations 16:1 (2000), 5-19. MR 2001g:34001 Zbl 0974.34005

[Collins 1997] C. B. Collins, "Conditions for a centre in a simple class of cubic systems", Differential Integral Equations 10:2 (1997), 333-356. MR 98c:34043 Zbl 0894.34022

[Conti 1994] R. Conti, "Uniformly isochronous centers of polynomial systems in $\mathbb{R}^{2}$," pp. 21-31 in Differential equations, dynamical systems, and control science, edited by K. D. Elworthy et al., Lecture Notes in Pure and Appl. Math. 152, Dekker, New York, 1994. MR 94i:34061 Zbl 0795.34021

[Dolov 1976] M. V. Dolov, "The canonical integral in the neighborhood of a focus”, Differencial'nye Uravnenija 12:11 (1976), 1946-1953. English translation: Differential Equations 12 (1977), 13621368. MR 56 \#738 Zbl 0376.34004

[Gasull and Llibre 1990] A. Gasull and J. Llibre, "Limit cycles for a class of Abel equations", SIAM J. Math. Anal. 21:5 (1990), 1235-1244. MR 91e:34036 Zbl 0732.34025

[Giacomini et al. 1996] H. Giacomini, J. Llibre, and M. Viano, "On the nonexistence, existence and uniqueness of limit cycles”, Nonlinearity 9:2 (1996), 501-516. MR 97a:34073 Zbl 0886.58087 
[Giné and Llibre 2004] J. Giné and J. Llibre, "Integrability and algebraic limit cycles for polynomial differential systems with homogeneous nonlinearities", J. Differential Equations 197:1 (2004), 147-161. MR 2004j:34077

[Giné and Santallusia 2001] J. Giné and X. Santallusia, "On the Poincaré-Lyapunov constants and the Poincaré series", Appl. Math. (Warsaw) 28:1 (2001), 17-30. MR 2002c:34044 Zbl 1022.34028

[Kamke 1943] E. Kamke, Differentialgleichungen: Lösungsmethoden und Lösungen, Mathematik und ihre Anwendungen 18, Becker \& Erler, Leipzig, 1943. Zbl 0028.22702

[Kooij and Christopher 1993] R. E. Kooij and C. J. Christopher, "Algebraic invariant curves and the integrability of polynomial systems", Applied Math. Letters 6:4 (1993), 51-53. MR 1348232 Zbl 0784.34008

[Llibre and Rodríguez 2004] J. Llibre and G. Rodríguez, "Configurations of limit cycles and planar polynomial vector fields", J. Differential Equations 198:2 (2004), 374-380. MR 2004j:34100

[Lloyd et al. 1997] N. G. Lloyd, C. J. Christopher, J. Devlin, J. M. Pearson, and N. Yasmin, "Quadratic-like cubic systems", Differential Eq. Dynam. Systems 5:3-4 (1997), 329-345. MR 99k:34061 Zbl 0898.34026

[Pearson et al. 1996] J. M. Pearson, N. G. Lloyd, and C. J. Christopher, "Algorithmic derivation of centre conditions", SIAM Rev. 38:4 (1996), 619-636. MR 98c:34050 Zbl 0876.34033

[Petit Bois 1961] G. Petit Bois, Tables of indefinite integrals, Dover, New York, 1961. MR 23 \#A256 Zbl 0098.26408

[Schlomiuk 1993] D. Schlomiuk, "Algebraic and geometric aspects of the theory of polynomial vector fields", pp. 429-467 in Bifurcations and periodic orbits of vector fields (Montreal, PQ, 1992), edited by D. Schlomiuk, NATO Adv. Sci. Inst. Ser. C Math. Phys. Sci. 408, Kluwer Acad. Publ., Dordrecht, 1993. MR 95a:34042 Zbl 0790.34031

[Singer 1992] M. F. Singer, "Liouvillian first integrals of differential equations", Trans. Amer. Math. Soc. 333:2 (1992), 673-688. MR 92m:12014 Zbl 0756.12006

[Volokitin 2002] E. P. Volokitin, "Center conditions for a simple class of quintic systems", Int. J. Math. Math. Sci. 29:11 (2002), 625-632. MR 2002m:34042 Zbl 1008.34023

Received April 11, 2003.

JAUME GINÉ

DEPARTAMENT DE MATEMÀTICA

UNIVERSITAT DE LLEIDA

AV. JAUME II, 69

25001 LLEIDA

SPAIN

gine@eup.udl.es

JAUME LLIBRE

Departament de MATEMÀtiques

UNIVERSITAT AUTÒNOMA DE BARCELONA

08193 - BELLATERRA, BARCELONA

SPAIN

jllibre@mat.uab.es 